Hautarzt 2012 - 63:823-823

DOI 10.1007/s00105-012-2432-x

Online publiziert: 7. Oktober 2012

(c) Springer-Verlag Berlin Heidelberg 2012

M. Magerl ${ }^{1}$ - J. Brasch ${ }^{2}$ - U. Förster ${ }^{3}$ - B. Hauswald ${ }^{4}$ - E.-B. Mohr ${ }^{2}$. J. Präßler ${ }^{5}$. R. Treudler ${ }^{6} \cdot$ R. Vetter $^{7} \cdot$ V. Wahn ${ }^{8} \cdot$ V. Zampeli $^{9} \cdot$ M. Ziemer $^{6} \cdot$ M. Maurer $^{1}$

${ }^{1}$ Klinik für Dermatologie, Venerologie und Allergologie, Allergie-

Centrum, Charité - Universitätsmedizin Berlin

${ }^{2}$ Universitäts-Hautklinik Kiel

${ }^{3}$ Hals-Nasen-Ohrenklinik, Charité Universitätsmedizin Berlin

${ }^{4}$ Klinik und Poliklinik für HNO, Universitätsklinikum Carl Gustav Carus,TU Dresden

${ }^{5}$ Klinik für Hautkrankheiten, Friedrich-Schiller-Universität, Jena

${ }^{6}$ Klinik für Dermatologie, Venerologie \& Allergologie, Universitätsklinikum Leipzig

${ }^{7}$ Universitätsklinik für Dermatologie und Venerologie, Magdeburg

${ }^{8}$ Klinik für Pädiatrie m. S. Pneumologie und Immunologie, Charité Universitätsmedizin Berlin

${ }^{9}$ Dermatologie, Venerologie und Allergologie, Immunologisches Zentrum, Städtisches Klinikum Dessau

\title{
Erratum zu: Diagnostik und Ausschluss des hereditären Angioödems
}

\section{Ein standardisierter Ansatz für die Praxis}

\section{Hautarzt (2012) 63:567-572 DOI 10.1007/s00105-012-2388-X}

${ }^{4}$ Klinik und Poliklinik für $\mathrm{HNO}$, Universitätsklinikum Carl Gustav Carus, TU Dresden

${ }^{5}$ Klinik für Hautkrankheiten, FriedrichSchiller-Universität, Jena

${ }^{6}$ Klinik für Dermatologie, Venerologie \& Allergologie, Universitätsklinikum Leipzig

${ }^{7}$ Universitätsklinik für Dermatologie und Venerologie, Magdeburg

${ }^{8}$ Klinik für Pädiatrie m. S. Pneumologie und Immunologie, Charité Universitätsmedizin Berlin

${ }^{9}$ Dermatologie, Venerologie und Allergologie, Immunologisches Zentrum, Städtisches Klinikum Dessau

\section{Korrespondenzadresse}

\section{Prof. M. Magerl}

Klinik für Dermatologie,

Venerologie und Allergologie, Allergie-Centrum, Charité - Universitätsmedizin Berlin

Charitéplatz 1, 10117 Berlin

Markus.Magerl@charite.de

${ }^{1}$ Klinik für Dermatologie, Venerologie und Allergologie, Allergie-Centrum, Charité - Universitätsmedizin Berlin

${ }^{2}$ Universitäts-Hautklinik Kiel

${ }^{3}$ Hals-Nasen-Ohrenklinik, Charité Universitätsmedizin Berlin 